\begin{tabular}{l|l|l|l}
$\begin{array}{c}\text { Case Reports in } \\
\text { NeUlology }\end{array}$ & $\begin{array}{l}\text { Case Rep Neurol 2011;3:14-17 } \\
\text { DOI: 10.1159/000323665 }\end{array}$ & $\begin{array}{l}\text { Published online: } \\
\text { January 14, 2011 }\end{array}$ & $\begin{array}{l}\text { O 2011 S. Karger AG, Basel } \\
\text { ISSN 1662-680X } \\
\text { www.karger.com/crn }\end{array}$ \\
\hline
\end{tabular}

\title{
Idiopathic Parkinson's Disease, Osteoporosis, and Hip Fractures: A Case Report
}

\author{
Richard A. Rison ${ }^{\mathrm{a}-\mathrm{d}}$ Kelsey Richardson ${ }^{\mathrm{a}-\mathrm{c}}$ \\ aUniversity of Southern California, ${ }^{b}$ Keck School of Medicine, and 'Los Angeles \\ County Medical Center, Los Angeles, Calif., and dPresbyterian Intercommunity \\ Hospital Stroke Center, Presbyterian Intercommunity Hospital, Whittier, \\ Calif., USA
}

\section{Key Words}

Idiopathic Parkinson's disease - Osteopenia - Osteoporosis - Hip fracture . Carbidopa-levodopa $\cdot$ L-dopa $\cdot$ Dyskinesias

\begin{abstract}
Patients with chronic idiopathic Parkinson's disease are at a high risk for fractures, particularly of the hip. The causes may be multifactorial, including poor balance and other forms of neurological dysfunction. Osteopenia and osteoporosis leading to decreased bone mass are common in these patients. We report a case of a male patient with a long-standing history of Parkinson's disease on chronic high-dose carbidopalevodopa who suffered a hip fracture. The relationship of Parkinson's disease and carbidopa-levodopa use with osteopenia and hip fractures is discussed, emphasizing the crucial need for prevention in this patient population along with medical and surgical treatment.
\end{abstract}

\section{Introduction}

Patients with idiopathic Parkinson's disease (IPD) are at a high risk for hip fractures. The causes may be multifactorial, including poor balance and other forms of neurological dysfunction. People with IPD also have a high frequency of osteopenia and osteoporosis leading to decreased bone mass compounding the risk of fracture. This short case report discusses various mechanisms and highlights the crucial need for prevention in these patients along with treatment.

\section{Case Presentation}

A 55-year-old Caucasian male had a 14-year history of IPD with worsening dyskinesias alternating with spells of immobility, postural instability, and 'wearing off phenomenon. He was in this usual state 
of baseline neurologic health when he arose from his computer one morning, stumbled and fell. Emergency medical services were activated and the patient was transported to the emergency department of our hospital where he was found to have a right hip fracture. The patient was seen by orthopedics and underwent a successful right hip arthroplasty without incident.

His past medical history was significant only for some depression and anxiety. There was no family history of IPD or any other progressive neurologic disorder. The patient had worked as a warehouse manager for a number of years until progression of his disease forced him to retire (about 5 years prior to his current presentation). There was no history of any illicit drug use. He did not smoke tobacco but reported alcohol use of 1 drink per month.

The patient had been on high doses of carbidopa-levodopa (25-250 mg, 1.5 tablets every $2 \mathrm{~h}$ while awake) and a dopamine agonist (ropinirole $2 \mathrm{mg}, 1$ tablet every $2 \mathrm{~h}$ while awake) for his rigidity and amantadine $(100 \mathrm{mg}, 1$ tablet every $5 \mathrm{~h}$ ) for his dyskinesias for a number of years.

Neurologic examination was remarkable for a patient in no acute distress. His right hip was in an immobilizer. He had prominent dyskinetic movements involving his arms, neck and face with hypophonic speech. Tone was increased in his bilateral upper extremities and finger-tapping amplitude was diminished. His mental status was quite intact, being able to recall objects and count backwards. No hallucinations or delusions were observed. No cranial nerve deficits were noted. Reflexes were intact at $2 / 4$ in the bilateral upper extremities and the left knee jerk. Motor strength testing was 5/5 (Medical Research Council Grade) in the bilateral upper extremities and the left lower extremity. Testing of the right lower extremity and gait was limited secondary to the post-operative hip immobilizer. There was no upper extremity dysmetria on finger-to-nose testing and sensation was intact to light touch, pain, and temperature throughout.

Written informed consent was obtained from the patient for publication of this case report.

\section{Discussion}

Parkinson's disease and osteoporosis are 2 disorders that affect a significant portion of patients. Patients with Parkinson's disease have a high risk of hip fractures. The causes may be multifactorial, including poor balance and other forms of neurological dysfunction including bradykinesia and hypokinesis leading to falls. Osteopenia and osteoporosis leading to decreased bone mass are also common in these patients [1].

Osteoporosis is more common in IPD patients than in age-matched controls. Up to $91 \%$ of females with IPD are found to have osteopenia or osteoporosis [1]. One of the main factors causing increased osteoporosis in these patients is decreased mobility leading to decreased weight-bearing exercise which is critical to maintain bone mass during aging. This lack of weight bearing causes increases bone reabsorption and decreases bone deposition. In addition, these patients are unstable and have an increased risk of falls. Besides bradykinesia and postural instability, both of which contribute to falls, autonomic instability further increases risk. It should be noted that autonomic instability may be a medication side effect - one that should be seriously considered before starting new medications in patients with IPD. One study has shown that dementia related to IPD increases fracture risk further, as these patients are less likely to be aware of their surroundings and physical limitations [2].

A 2007 study showed that levodopa is associated with an increased fracture risk, and that the risk is dose dependent. This correlation was greatest when analyzing hip fracture specifically [3]. Some believe that the increased fracture risk is due to the fact that levodopa increases patient mobility without improving postural stability, providing 
increased opportunity for fall and fracture [4]. It has also been shown that anti-IPD medications, specifically L-dopa, increase serum homocysteine levels [5]. An elevated homocysteine level is an independent risk factor for fracture, regardless of the patient's IPD status.

Although IPD is a serious, progressive illness, one must not forget other clinical factors applicable to each individual patient that may change their risk stratification for fractures. Like all patients, increased age, female gender, low body mass, and vitamin D deficiency all increase fracture risk [1]. Any patient may have a variety of these risk factors along with the added factors associated with IPD.

Because of the often debilitating nature of hip fractures, clinicians should do whatever possible to prevent fractures in the first place. It has been shown that vitamin D supplementation and bisphosphonate treatment can decrease fracture risk [1]. However, other studies show no statistically significant difference between those treated prophylactically with risedronate versus placebo [6]. It is important to note that even in this trial, where fracture risk was not greatly affected, bone mineral density was significantly better in the bisphosphonate group. Urine markers of bone resorption were also significantly improved, signifying a role for bisphosphonate prophylaxis in these patients. This study also included daily ergocalciferol treatment in both groups - which may help counter bone resorption in these patients.

Regardless of the cause of hip fracture in IPD patients, these fractures do occur and require vigilant management. Many studies have postulated that these patients have an increased mortality when compared to controls without IPD; however, these studies have had conflicting results [4]. Initial clinical parameters influence a patient's clinical course, such as age, gender, mobility, etc. From the orthopedic prospective, it is difficult to compare outcomes of IPD patients with hip fractures from other patients with hip fractures. Some studies suggest that 6-month mortality is similar for both groups, around $14 \%$, where other studies suggest IPD mortality is closer to $50 \%$ at 6 months [4]. Controversy also exists regarding how these fractures should be treated. Internal fixation appears to be a good option for patients with IPD; however, this is not always feasible when patients have a higher grade fracture. It is thought that endoprostheses for severe fractures are associated with poor outcomes in IPD because they delay mobility postoperatively.

Overall, this review of the literature illustrates that hip fractures are a large problem in the population of patients with IPD. It is important to counsel patients and family members about these risks, so they are aware of how they can prevent fractures in their home. In addition, it is an important issue for neurologists, orthopedists and primary care physicians to be aware of when seeing patients with IPD. It appears that controversy still exists regarding the best method of management for these fractures as well as long-term outcome. Although it is crucial for orthopedists dealing with hip fractures to learn the best course of treatment, even more emphasis should be placed on prevention.

\section{Conclusion}

Patients with IPD have a high risk of osteopenia and hip fractures. The causes are multifactorial. Treatment exists, but prevention is paramount. 


\section{Disclosure Statement}

R.A.R. serves as Associate Neurology Editor for 'Journal of Medical Case Reports', 'Case Reports in Neurology', 'Grand Rounds', and formerly 'Cases Journals'. K.R. has nothing to disclose.

\section{References}

1 Invernizzi M, Carda S, Viscontini GS, Cisari C: Osteoporosis in Parkinson's Disease. Parkinsonism Relat Disord 2009;15:339-346.

$\checkmark 2$ Melton LJ 3rd, Leibson CL, et al: Fracture risk after the diagnosis of Parkinson's disease: Influence of concomitant dementia. Mov Disord 2006;21:1361-1367.

3 Vestergaard P, Rejnmark L, Mosekilde L: Fracture risk associated with parkinsonism and anti-Parkinson drugs. Calcif Tissue Int 2007;81:153-161.

4 Clubb VJ, Clubb SE, Buckley S: Parkinson's disease patients who fracture their neck of femur: a review of outcome data. Injury 2006;37:929-934.

5 Miller JW, et al: Effect of L-dopa on plasma homocysteine in PD patients: relationship to B vitamin status. Neurology 2003;60:1125-1129.

6 Sato Y, Honda Y, Iwamoto J: Risedronate and ergocalciferol prevent hip fracture in elderly men with Parkinson disease. Neurology 2007;68:911-915. 